\title{
LIMITATION OF BEAM CURRENT IN THE PLS STORAGE RING
}

\author{
E.-S. Kim *, M.-H. Chun, J.-Y. Huang, U.-H. Huang, H.-S. Kang, \\ D.-T. Kim, S.-H. Nam, H.-J. Park, J.-S Yang, M. Yoon and I.-H. Yu \\ Pohang Accelerator Lab., POSTECH, Pohang, KyungBuk, 790-784, Korea
}

\begin{abstract}
We present investigations on beam instabilities that have been observed at $2.0 \mathrm{GeV}$ and $2.5 \mathrm{GeV}$ in the PLS storage ring. Stored beam currents of $2.0 \mathrm{GeV}$ in the ring has been mainly limited by coupled-bunch instabilities. We have investigated dependences of the coupled-bunch instabilities on betatron tune, chromaticity and rf cavity temperatures at $2.0 \mathrm{GeV} .450 \mathrm{~mA}$ beam could be stored at $2.0 \mathrm{GeV}$. Bunch filling patterns and betatron tune are investigated to suppress the beam instabilities at $2.5 \mathrm{GeV}$. At $2.5 \mathrm{GeV}$, we do not observe any beam instabilities up to $200 \mathrm{~mA}$. Higher beam currents than $200 \mathrm{~mA}$ are limited by total $\mathrm{rf}$ power.
\end{abstract}

\section{INTRODUCTION}

The PLS storage ring is a third generation synchrotron radiation source. It consists of a $2 \mathrm{GeV}$ linac and a storage ring which can be accelerated by $2.0 \mathrm{GeV}$ to $2.5 \mathrm{GeV}$. The storage ring has been operated at $2.0 \mathrm{GeV}$ from 1995 to 1999 and at $2.5 \mathrm{GeV}$ since 2000 . The parameters of the storage ring are listed in Table 1.

It is a general requirement on the storage ring for a synchrotron radiation source that it provides a stable beam having a small emittance in order to obtain a high brilliance photon beam. It is apparent that the beam quality is strongly determined by beam instabilties in such a machine having a low emittance. Therefore, a study to cure beam instabilities is important to obtain a stable and small beam [1]. The designed value of the beam current in the PLS storage ring is $400 \mathrm{~mA}$ at $2.0 \mathrm{GeV}$. However, PLS storage ring has operated in less beam currents than 190 $\mathrm{mA}$ at an energy of $2.0 \mathrm{GeV}$ which has been limited by the coupled-bunch instabilities. The machine study at the PLS has been focused to cure the beam instabilities and to store high beam current. In this paper, we present the activities to suppress the beam instabilities at $2.0 \mathrm{GeV}$ and $2.5 \mathrm{GeV}$ in the PLS storage ring during the last one year.

\section{BEAM INSTABILITIES AND CURRENT LIMITATION AT 2.5 GEV}

The storage ring has been operated at $170 \mathrm{~mA}$ of 2.5 GeV since January 2000. During the user operation between January 2000 and July 2000, the number of bunches was 468 that was equal to the harmonic number. Operated tune was 14.26 and 8.15 in horizontally and vertically, respectively. We observed resonant frequency of $831.8 \mathrm{MHz}$ in beam spectrum due to higher order mode (HOM) in $\mathrm{rf}$

\footnotetext{
*eskim1@ postech.ac.kr
}

cavities. Figure 1(a) shows the beam oscillation that is observed in streak camera. We see that the beam motions are unstable.

Since September 2000, we have changed the number of bunches and betatron tune for the user operation. The number of bunches is 400 and operating tune is 14.28 and 8.18 in horizontally and vertically, respectively. Figure 1(b) shows the beam oscillation that is observed in streak camera. We see that the beam motions become more stably. We don't observe resonant frequency in beam spectrum due to rf HOMs. Deformed beam shape were not observed in beam profile monitor. Vacuum value in the ring is $0.6 \mathrm{nTorr}$ in the beam current of $170 \mathrm{~mA}$ in 400 bunches. At $2.5 \mathrm{GeV}$ we can store the beam stably up to $200 \mathrm{~mA}$. Higher beam current than $200 \mathrm{~mA}$ in present operation is limited by total rf power. The beam lifetime is about 26 hours in $170 \mathrm{~mA}$.

\section{BEAM INSTABILITIES AND CURRENT LIMITATION AT 2.0 GEV}

\subsection{Machine study in April 2000}

We have experienced coupled-bunch instability over the beam current of $200 \mathrm{~mA}$. We observed that beam was an elliptic under around $200 \mathrm{~mA}$ and the shape suddenly expanded to the horizontal direction over $200 \mathrm{~mA}$. Transverse $834 \mathrm{MHz}$ rf HOM was observed in the beam spectrum. We increased currents of SD (vertical focusing) and SF (horizontal focusing) sextupoles by $10 \%$. Then it was observed that the HOM was suppressed. Beam was injected again and the beam with elliptic shape was obtained up to 200 $\mathrm{mA}$. But, long injection time of 30 minutes for $200 \mathrm{~mA}$ was taken due to changed betatron tune.

Above $200 \mathrm{~mA}$, longitudinal $758 \mathrm{MHz}$ rf HOM was observed in \# 1 cavity. Then elliptic beam was changed to longitudinally deformed beam. To suppress the instability, we changed water temperture of rf cavity \# 1 at the rate of 0.5 degree. When the beam gradually expands, we reversed the direction of variation in temperature. It means that we need to measure HOM's frequencies about all cavities beforehand. Then the $758 \mathrm{MHz}$ rf HOM could be suppressed and the beam shape was kept up to about $250 \mathrm{~mA}$.

About $250 \mathrm{~mA}$, the information obtained by spectrum analyzer showed that no HOM's frequencies were observed, but the beam was shaken. Then we changed the currents of SD and SF sextupoles to lower or higher values in order to obtain stable beam as we observe the beam shape in beam profile monitor. After this procedure, the deformation of beam was cured and the beam became an elliptic shape. More $30 \mathrm{~mA}$ beam could be stored maintaining stable beam. 
In $280 \mathrm{~mA}$, we detuned rf cavity \# 1 and \# 4 by $1.5 \mathrm{kHz}$ more. More $20 \mathrm{~mA}$ could be stored by detuning the fundamental frequency. At last we could store $300 \mathrm{~mA}$ beam for just 20 minutes. On the other hand, vacuum value inside rf cavities was increased to $10^{-7}$ Torr in higher beam current than $280 \mathrm{~mA}$.

In resut, to store $300 \mathrm{~mA}$ at $2 \mathrm{GeV}$ in the storage ring, we performed activities such as the detuning of cavity frequency to remove Robinson instability, temperature control of the rf cavity to suppress HOMs and the suppression of transverse instability by control of chromaticity.

\subsection{Machine study in May 2000}

In 5 May, we could store the beam stably up to $250 \mathrm{~mA}$ with $\mathrm{SD}=135 \mathrm{~A}$ and $\mathrm{SF}=90 \mathrm{~A}$. Above $250 \mathrm{~mA}$, we changed the currents of sextupoles to lower or higher values as we observe the beam shape in beam profile monitor in order to obtain stable beam. Then we could store $300 \mathrm{~mA}$ beam stably for 10 hours. Figure 2 shows the stored beam current of $300 \mathrm{~mA}$. Betatron tune was $\nu_{x}=14.28$ and $\nu_{y}=8.18$.

\subsection{Machine study in June 2000}

Betatron tune survey was performed to understand the characteristics of the transverse rf HOMs. We also investigated effects of bunch filling patterns on the beam instability. Figure 3 shows the beam spectra in $175 \mathrm{~mA}$ of 2.0 GeV. Fig.3(a) shows the beam spectrum for the case of 468 bunches, $\nu_{x}=14.26$ and $\nu_{y}=18.16$. Fig.3(b) shows the beam spectrum for the case of 468 bunches, $\nu_{x}=14.20$ and $\nu_{y}=18.19$. Fig.3(c)shows the beam spectrum for the case of 400 bunches, $\nu_{x}=14.26$ and $\nu_{y}=18.19$. Here, we note that positions and magnitudes of rf HOMs are affected by the number of bunches and betatron tunes. Figure 4 shows the increased magnitude of vertical tune frequency for the case of 468 bunches. It seemed to be related to ion effect. The increased magnitude of vertical tune frequency could be greatly decreased for the case of 400 bunches.

\subsection{Machine study in November 2000}

We attempted to store the beam current as high as possible at a fixed tune. Horizontal coupled-bunch instability was observed during beam injection and storage. As the stored beam current was increased, horizontal beam blowup was seen on the beam profile monitor. It was observed that the horizontal-coupled instability due to $830.45 \mathrm{MHz}$ HOM drops the stored beam around $250 \mathrm{~mA}$. Then, vacuum value inside rf cavities was also increased to above 100 nTorr. At last, the beam was suddenly dropped around $250 \mathrm{~mA}$. To shift the transverse HOM, we selected the betatron tune of $\nu_{x}=14.27$ and $\nu_{y}=8.23$ that suppresses the transverse rf HOM. In this process the frequency spectrum of the beam was monitored to confirm that the instability was always related with the horizontal coupled-bunch oscillation. It was observed with spectrum analyzer that the resonant frequency was $830.45 \mathrm{MHz}$. The tune dependence of the instability was studied for a wider range. To suppress the transverse instability such as head-tail instability, we also adjusted the chromaticity. Contrary to the May and March cases, we fixed the betatron tune and chromaticity during the beam injection. We could suppress the transverse instabilities and could store $430 \mathrm{~mA}$ beam, without showing increased vacuum value inside rf cavities. Above $400 \mathrm{~mA}$ we observed the longitudinal 758.6 MHz rf HOM. But, the longitudinal coupled-bunch instabilities modes did not lead to beam loss up to $430 \mathrm{~mA}$.

\subsection{Machine study in April 2001}

In April 2001, we choosed a different optimal betatron tune with November 2000 by tune survey. The selected tune was $\nu_{x}=14.19$ and $\nu_{y}=8.25$. We did not change betatron tune and chromaticity during the beam injection. We did not observe the transverse rf HOM and horizontal beam blow-up, and the beam could be stored up to $450 \mathrm{~mA}$, without showing increased vacuum value inside rf cavities. Above $400 \mathrm{~mA}$ we observed the longitudinal 758.6 MHz rf HOM. The longitudinal coupled-bunch instability mode does not lead to beam loss up to $450 \mathrm{~mA}$. We did not store higher beam current than $450 \mathrm{~mA}$ for the safety of vacuum components. The injection rate was typically 2-3 mA per pulse and showed better injection rate than case of November 2000. Figure 5 shows the stored beam current of 450 $\mathrm{mA}$. The beam lifetime at the $450 \mathrm{~mA}$ was 6 hours.

\section{BEAM INSTABILITIES TO BE CURED MORE}

\subsection{Longitudinal-coupled bunch instability}

At this moment, we still observe longitudinal coupledbunch instabilities around $360 \mathrm{~mA}$ due to $758.66 \mathrm{MHz}$ and $1300 \mathrm{MHz}$ modes at $2.0 \mathrm{GeV}$. The instability due to the $1300 \mathrm{MHz}$ mode does not lead to beam loss since the shunt impedance of the mode is small. The $1300 \mathrm{MHz}$ mode disappeare as the stored beam current increases. The instability due to the $758.66 \mathrm{MHz}$ mode does not lead to beam loss up to $450 \mathrm{~mA}$. However, the longitudinal beam oscillation due to the $758.6 \mathrm{MHz}$ mode enlarges beam size horizontally, and moreover, accompanies with beam size fluctuation and bunch-lengthening. When the fluctuation amplitude due to the $758.66 \mathrm{MHz}$ mode is large, it is observed that the beam lifetime decreases.

\section{CONCLUSION}

In this paper, we present the activities to suppress the beam instabilities at $2.0 \mathrm{GeV}$ and $2.5 \mathrm{GeV}$ in the PLS storage ring during the last one year. We have investigated dependences of the beam instabilities on betatron tune, chromaticity, bunch filling patterns and rf cavity temperatures. The designed beam current at $2.0 \mathrm{GeV}$ in the ring could be stored. Longitudinal coupled-bunch instability due to 758.6 $\mathrm{MHz}$ mode at $2.5 \mathrm{GeV}$ remains in only instability 
Table 1: Designed parameters in the PLS storage ring

\begin{tabular}{|l|c|c|}
\hline Parameters & $\mathbf{2 . 0 ~ G e V}$ & $\mathbf{2 . 5} \mathbf{~ G e V}$ \\
\hline Lattics type & TBA & TBA \\
Circumference & $280.56 \mathrm{~m}$ & $280.56 \mathrm{~m}$ \\
Natural emittance & $12.1 \mathrm{~nm}$ & $18.9 \mathrm{~nm}$ \\
Harmonic number & 468 & 468 \\
Energy spread & 0.00068 & 0.00085 \\
Synchrotron frequency & $11.7 \mathrm{kHz}$ & $10 \mathrm{kHz}$ \\
RF voltage & $1.6 \mathrm{MV}$ & $1.6 \mathrm{MV}$ \\
Damping time(T/L) & $16.6 / 8.3 \mathrm{~ms}$ & $8.5 / 4.2 \mathrm{~ms}$ \\
Bunch length & $5 \mathrm{~mm}$ & $8 \mathrm{~mm}$ \\
\hline
\end{tabular}

mode to be cured in the ring. We do not observe beam instabilities at $2.5 \mathrm{GeV}$ up to $200 \mathrm{~mA}$. Higher beam currents than $200 \mathrm{~mA}$ at $2.5 \mathrm{GeV}$ are limited by the total $\mathrm{rf}$ power.

\section{REFERENCES}

[1] A.W. Chao, Physics of Collective Beam Instabilities in High Energy Accelerators ( Wiley, New York, 1993). (a)

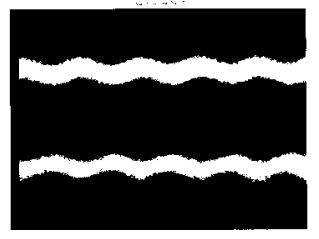

(b)

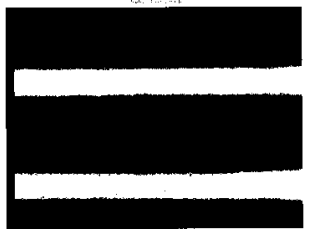

Figure 1: Beam oscillations at $2.5 \mathrm{GeV}$ that are observed in streak camera. (a) the number of bunches is $468, \nu_{x}=14.26$ and $\nu_{y}=8.15$. (b) the number of bunches is $400, \nu_{x}=14.28$ and $\nu_{y}=8.18$. Beam currents in (a) and (b) are $115 \mathrm{~mA}$ and $110 \mathrm{~mA}$, respectively.

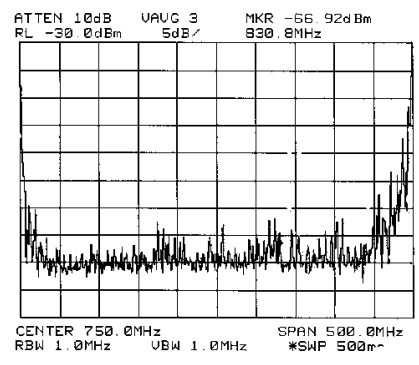

Figure 2: Beam spectrum on spectrum analyzer in the beam current of $193 \mathrm{~mA}$ at $2.5 \mathrm{GeV}$. The spectrum shows frequency band between $500 \mathrm{MHz}$ and $1 \mathrm{GHz}$.

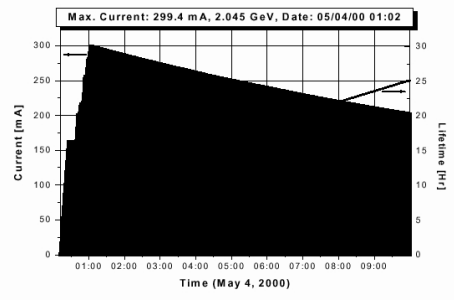

Figure 3: Stored beam current of $300 \mathrm{~mA}$ at $2.0 \mathrm{GeV}$. (a)

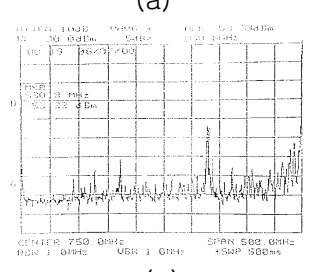

(c)

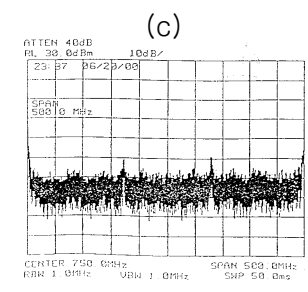

(b)

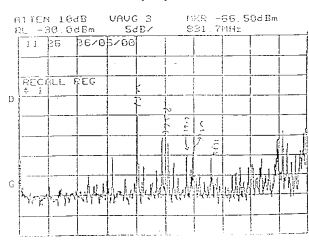

)

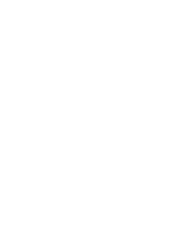

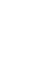

Figure 4: Beam spectra at $2.0 \mathrm{GeV}$ that show frequency bands between $500 \mathrm{MHz}$ and $1 \mathrm{GHz}$. (a) 468 bunches, $\nu_{x}=14.26$ and $\nu_{y}=18.16$. (b) 468 bunches, $\nu_{x}=14.20$ and $\nu_{y}=18.19$. (c) 400 bunches, $\nu_{x}=14.26$ and $\nu_{y}=18.19$.

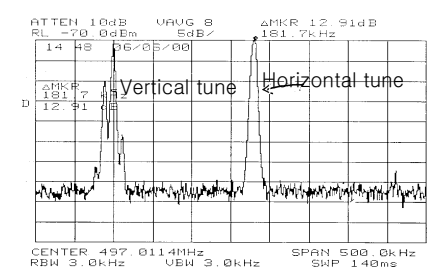

Figure 5: Beam spectrum shows increased magnitude and side-bands in vertical tune when number of bunches is 468 .

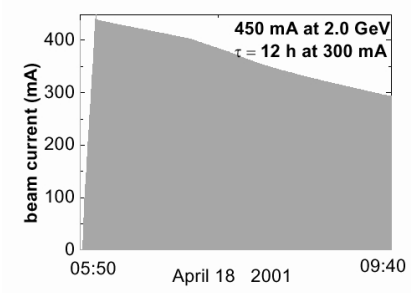

Figure 6: Stored beam current of $450 \mathrm{~mA}$ at $2.0 \mathrm{GeV}$. 